\title{
UGT1A1*28 Genotypes and Respiratory Disease in Very Preterm Infants: A Cohort Study
}

\author{
Jesper Padkær Petersen ${ }^{a, b}$ Finn Ebbesen ${ }^{b} \quad$ Mads Vilhelm Hollegaard $^{d}$ \\ Sofia Andersson $^{b}$ David Michael Hougaard ${ }^{d}$ Ole Thorlacius-Ussing ${ }^{c}$ \\ Tine Brink Henriksen ${ }^{a}$ \\ a Department of Pediatrics, Aarhus University Hospital, Aarhus, Departments of b Pediatrics and 'S Surgery, \\ Aalborg University Hospital, Aalborg, and ${ }^{\mathrm{d}}$ Statens Serum Institut, Danish Centre for Neonatal Screening, \\ Copenhagen, Denmark
}

\section{Key Words}

Bilirubin - Bronchopulmonary dysplasia . Preterm infant .

Antioxidants - UGT1A $1 * 28$ allele

\begin{abstract}
Background: Respiratory disease in the very preterm infant is frequent and often severe. Bilirubin is both a potent neurotoxin and antioxidant, and may have a clinical impact on preterm respiratory disease. The Gilbert genotype, the UGT1A $1 * 28$ allele, is the major known genetic cause of variation in bilirubin. Objectives: To study the association between respiratory disease in the very preterm infant and the UGT1A $1 * 28$ allele. Methods: This is a cohort study of 1,354 very preterm infants (gestational age $<32$ weeks) born in Jutland, Denmark in 1997-2011. Genotypes were obtained from the Danish Neonatal Screening Biobank, and clinical information was obtained from the databases of two tertiary neonatal intensive care units. Outcomes were the need for surfactant therapy, any need for and duration of supplementary oxygen and bronchopulmonary dysplasia (BPD). Results: Per UGT1A $1 * 28$ allele, odds were increased for any need of supplementary oxygen (odds ratio 1.26; 1.05-1.50) and for BPD (odds ratio $1.71 ; 1.23-2.39$ ), the need of supplementary oxygen increased by 6.38 days (1.87-10.89), and
\end{abstract}

\section{KARGER}

E-Mail karger@karger.com www.karger.com/neo

\section{(c) 2015 The Author(s) \\ Published by S. Karger AG, Basel 1661-7800/15/1092-0124\$39.50/0}

This article is licensed under the Creative Commons AttributionNonCommercial-NoDerivatives 4.0 International License (CC BYNC-ND) (http://www.karger.com/Services/OpenAccessLicense) Usage and distribution for commercial purposes as well as any distribution of modified material requires written permission. chance per day of no longer needing supplementary oxygen was reduced (hazard rate $0.84 ; 0.76-0.93$ ). No effect was observed for need of surfactant treatment (odds ratio 1.08; 0.91-1.28). Hardy-Weinberg equilibrium was unlikely for the cohort $(p<0.012)$. This could be explained by death prior to genotype sampling. In tests of robustness this failed to explain the primary results. Conclusions: Compared to the common genotype, UGT1 A $1 * 28$ genotypes were associated with an increased need of oxygen supplementation and risk of BPD in very preterm newborns. @2015 The Author(s)

Published by S. Karger AG, Basel

\section{Introduction}

Oxidative stress is thought to be involved in respiratory disease amongst preterm infants, and antioxidants may accordingly be important in its prevention $[1,2]$.

Bilirubin is neurotoxic, but it is also an antioxidant of possible clinical relevance [3]. The association between bilirubin and respiratory disease in the preterm population has been explored in previous observational studies and as a secondary outcome in an interventional study, with diverging results [4-6]. The challenges in noninterventional studies of bilirubin's influence on neonatal out- 
comes are the possibility of reverse causality and the presence of potential confounding factors. One way of addressing these challenges is by utilizing the genotype of Gilbert-Meulengracht syndrome, the UGT1A $1 * 28$ allele [7].

The rate-limiting enzyme in the metabolism of bilirubin is hepatic uridine diphosphate glucuronosyltransferase 1A1 (UGT1A1) [8]. In the UGT1A1*28 allele an extra TA pair is inserted in the TATA box of the UGT1A1 gene, reducing the activity of hepatic UGT1A1 by approximately $30 \%$ per allele. The allele is the major known genetic cause of variation in plasma bilirubin in adult Western European populations, accounting for approximately $18 \%$ of all variations [ $8-10]$. In the term neonatal population, the allele has been associated with accelerated development and duration of hyperbilirubinemia, while the association with crossing of treatment thresholds is uncertain [11-13]. No data exist for the preterm population.

Mendel's second law states that alleles are inherited randomly at conception. As genotypes are permanent, estimates of the association between UGT1A $1 * 28$ genotypes and respiratory outcomes will be unconfounded, and thus the possibility of reverse causality is eliminated $[9,14]$. To test the effect of genetically elevated bilirubin, we investigated the association between UGT1A1*28 genotypes and respiratory disease in a cohort of 1,354 very preterm infants.

\section{Subjects and Methods}

The study setting was two regions in Jutland, Denmark (19972011), with a population of 1.3 million people and some 21,000 live births per year. The study units were the two regional tertiary neonatal intensive care units (NICUs).

In Denmark, the first choice of respiratory support is nasal continuous positive airway pressure, with surfactant provided as rescue treatment to preterm infants with respiratory distress syndrome, according to nationally uniform criteria as described by Verder et al. [15]. During the study period, the two units used supplementary oxygen, targeting saturations at $88-92 \%$.

The neonatal database of the two tertiary NICUs provided data on birth weight, gestational age (best obstetric estimate), date of last day with oxygen supplementation (if any) and date of discharge [16]. The database does not hold information on bilirubin measures or phototherapy. The study population was restricted to primary admissions at a study NICU within the first $24 \mathrm{~h}$ of life, gestational age $<32$ weeks, birth weight $<2,000 \mathrm{~g}$ and no life-threatening malformations. Follow-up ended at first discharge from the tertiary NICU or death.

At birth, Danish citizens are assigned a unique 10-digit civil registration $(\mathrm{CPR})$ number, which allows unambiguous individual-level identification and data linkage across nationwide registers and hospital records [17]. The parent's country of birth and the present status of the child (alive, dead or emigrated, and date of death if relevant) were obtained from the Danish CPR register [17]. The date of birth and sex of the infant can be unambiguously derived from the CPR number.

As part of the Danish national neonatal screening program for metabolic diseases, a dried blood spot sample is collected routinely from all newborns shortly after birth. The excess dried blood spot material is stored in the Danish Neonatal Screening Biobank, which covers about $99 \%$ of all newborns [18]. In the study units, dried blood spot sampling amongst the very preterm was delayed until 28 days' postnatal age for most of the study period, and therefore preterm nonsurvivors are rarely present in the Biobank.

The dried blood spots of the cohort were identified in the Biobank by the use of the CPR numbers. DNA was extracted and subsequently whole-genome amplified, as previously described [19]. Genotyping was performed at LGC Genomics (Teddington, UK) using a custom-made competitive allele-specific PCR genotyping assay. Genotyping was executed jointly with two other studies (total $n=4,147$ ), and the laboratory was unaware of individual sample identities, project belongings and the type and the aim of the projects. In a subsample of the studies ( $n=648)$, genotyping was also performed at Statens Serum Institut (Copenhagen, Denmark), using the pyrosequencing platform (Qiagen, Hilden, Germany) [20]. The concordance rate between the genotypes produced by competitive allele-specific PCR compared to pyrosequencing was $99.8 \%$.

\section{Statistical Methods}

We used STATA version 12.1 (StataCorp, College Station, Tex., USA) for statistical analyses. In the primary analyses, UGT1A $1 * 28$ genotypes were treated as a continuous variable (common genotype $=0$, heterozygotes $=1$, homozygotes $=2$ ) and in secondary analyses as a categorical variable in three groups, with common genotype as the reference.

Dichotomous estimates of respiratory disease were any need of additional oxygen, need of surfactant therapy and bronchopulmonary dysplasia (BPD) defined as the need of supplemental oxygen at 36 postmenstrual weeks. Infants who left the study prior to this age were included as not having BPD if supplementary oxygen was discontinued at least 1 day prior to discharge; infants leaving the study with oxygen supplementation prior to 36 postmenstrual weeks' gestational age were excluded. Dichotomous associations are reported as odds ratios (OR) from logistic regression analysis.

The continuous variable related to respiratory disease was time with supplementary oxygen (days) and was estimated as hazard ratios (HR) for discontinuation of supplementary oxygen using Cox regression analysis, and the absolute difference in the number of days with supplementary oxygen was estimated using generalized linear methods with pseudo values [21]. The time scale was days from birth, and the event was discontinuation of supplementary oxygen. Censoring occurred if the infant left the unit or died.

In all analyses, siblings were treated as clusters based on the maternal CPR number. All analyses were adjusted for African heritage as a continuous covariate (parent born in Africa: no $=0$, one $=1$, both $=2$ ), sex and year of birth as a categorical covariate (1997-2001, 2002-2006 and 2007-2011).

Hardy-Weinberg equilibrium (HWE) was estimated using the ' $h w s n p$ command'. Spearman's rank correlation was used to evaluate genotype distributions across four gestational age groups (2425 weeks, 26-27 weeks, 28-29 weeks and 30-31 weeks). 
Table 1. Basic clinical characteristics of 1,354 consecutive very preterm infants with UGT1A1 genotyping from the Mid and Northern Regions of Jutland, Denmark, 1996-2011

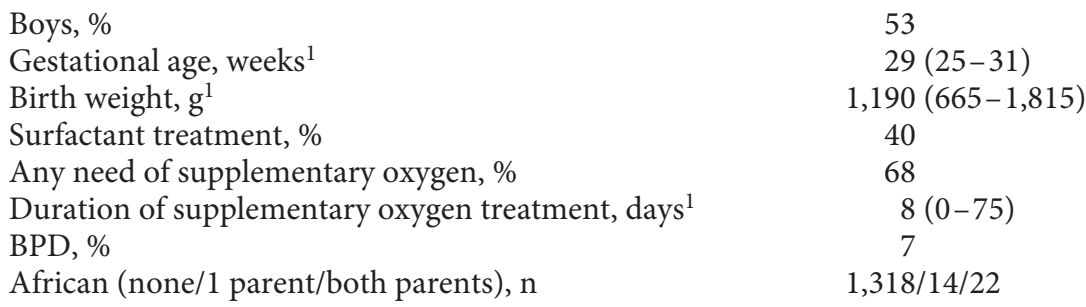

Boys, \%

Gestational age, weeks ${ }^{1}$

Birth weight, $\mathrm{g}^{1}$

Surfactant treatment, \%

Any need of supplementary oxygen, \%

Duration of supplementary oxygen treatment, days ${ }^{1}$

BPD, \%

African (none/1 parent/both parents), $\mathrm{n}$

$1,318 / 14 / 22$

BPD is defined as the need of supplementary oxygen at 36 weeks' postmenstrual age.

${ }^{1}$ Data are presented as medians (with 5-95 percentile range).
Death and BPD/discontinuation of supplementary oxygen were competing outcomes, and the majority of infants who died were not in the Biobank. As this could potentially cause immortal person-time bias in our study, we performed two tests of robustness for primary results. Continuous outcomes were re-examined with the use of a time scale of days from 28 days' postnatal age, the routine day of metabolic screening for very preterm infants in the two NICUs and thus the presumed day of study entry. Furthermore, all analyses were repeated and stratified into infants with a high mortality and low mortality, corresponding to gestational ages of 24-28 weeks and 29-31 weeks. All estimates are reported with $95 \%$ confidence intervals.

\section{Ethics}

The study was approved by the regional Ethics Committees on Human Studies (J.nr. 1-10-72-363-12), the Danish Data Protection Agency (J.nr. 1-16-02-153-12) and the Danish Neonatal Biobank Steering Committee, and conducted according to the principles of the Declaration of Helsinki.

\section{Results}

The 1,773 infants who fulfilled the criteria for study entry were identified in the neonatal database. For 1,354 of these, the genotype and information on supplementary oxygen were available. A total of 159 infants died before the 28-day postnatal cutoff, and a genotype was not available in 132 of these infants.

The basic characteristics of the study cohort are shown in table 1 . Of the 1,354 infants, 920 received supplementary oxygen for at least 1 day. Per UGT1A1*28 allele, we found an unadjusted increase in odds for any need of supplementary oxygen of $24 \%$ (OR $1.24 ; 1.04-1.48$ ), for surfactant treatment of $10 \%$ (OR 1.10;0.93-1.30) and for BPD of $73 \%$ (OR 1.73; 1.24-2.40). Per allele, the chance of being weaned off oxygen at any given day was $16 \%$ lower (HR 0.84; 0.76-0.93), and the need of supplementary oxygen increased by 6.49 days (2.1-10.9). Adjustment for infant sex, year of birth and African ethnicity had almost no effect on the estimates (table 2). Analyses of genotype as a categorical variable gave similar results (data not shown).

HWE was unlikely for the study cohort $(\mathrm{p}<0.012)$ and also for the part of the cohort in which both parents were born in Denmark $(\mathrm{p}<0.005)$. To assess whether excess mortality prior to dried blood spot sampling may explain some of the deviation from HWE, we used gestational age in four groups as a proxy variable for likelihood of death. UGT1A $1 * 28$ genotypes were more frequent in the high mortality/low gestational age stratums, and this distribution was unlikely to be caused by chance ( $<<0.005$; table 3 ).

The association between genotype and respiratory support stratified by a proxy variable for high and low mortality (24-28 weeks' gestational age and 29-31 weeks' gestational age) was estimated (online suppl. material; see www.karger.com/doi/10.1159/000442042). Overall, the estimates were smaller for all respiratory outcomes in the higher gestational age/low mortality stratum. However, the results between the two strata were quite similar.

Of the original 1,773 infants in the cohort, 1,614 were alive at 28 postnatal days. Data on genotype and oxygen treatment were available in 1,343 of these, of whom 366 received oxygen at that time and thus could enter the analysis of continuous outcomes. Seventeen infants died after 28 days, but in only 3 of these was the dried blood spot missing.

With days from the 28th day after birth as the time frame, we found (unadjusted per allele) that the chance of being weaned from oxygen any day decreased by $14 \%$ (HR $0.86 ; 0.73-1.00)$ and that 4.98 extra days of extra oxygen was needed (-0.63 to 10.58). Again, adjustment for infant sex, year of birth and African ethnicity left estimates almost unchanged. Fully adjusted estimates for these analyses for UGT1A $1 * 28$ heterozygotes and homozygotes are given in table 4 . When genotype was considered a categorical variable, the results were similar (data not shown). 
Table 2. The association between UGT1A1*28 genotypes and respiratory morbidity for 1,354 very preterm infants

\begin{tabular}{llllll}
\hline & $\begin{array}{l}\text { Surfactant } \\
\text { treatment, } \\
\text { OR }(95 \% \mathrm{CI})\end{array}$ & $\begin{array}{l}\text { Any need of } \\
\text { oxygen, } \\
\text { OR }(95 \% \mathrm{CI})\end{array}$ & $\begin{array}{l}\text { Cessation of supple- } \\
\text { mentary oxygen, } \\
\text { HR }(95 \% \mathrm{CI})\end{array}$ & $\begin{array}{l}\text { Duration of oxygen } \\
\text { treatment, } \\
\text { difference days }(95 \% \mathrm{CI})\end{array}$ & $\begin{array}{l}\text { BPD, } \\
\text { OR (95\% CI) }\end{array}$ \\
\hline Common genotype & Ref. & Ref. & Ref. & Ref. & Ref. \\
UGT1A ${ }^{*} 28$ heterozygous & $1.08(0.91-1.28)$ & $1.25(1.05-1.50)$ & $0.84(0.76-0.93)$ & $6.38(1.87-10.89)$ & $1.71(1.23-2.39)$ \\
UGT1A1 ${ }^{*} 28$ homozygous & $1.17(0.83-1.64)$ & $1.57(1.10-2.24)$ & $0.71(0.58-0.87)$ & $12.75(3.73-21.78)$ & $2.93(1.51-5.70)$ \\
\hline
\end{tabular}

All analyses were adjusted for sex, birth year and African race. OR were estimated by conditional logistic regression, HR by Cox regression analysis and numeric difference in days with oxygen by generalized linear methods with pseudo values. BPD is defined as the need of supplementary oxygen at 36 weeks' postmenstrual age.

Table 3. Distribution of UGT1A1*28 genotypes and neonatal mortality on 4 gestational age strata for a cohort of very preterm infants

\begin{tabular}{|c|c|c|c|c|}
\hline $26-27$ & $43.5(128)$ & $42.2(124)$ & $14.3(42)$ & 14.9 \\
\hline $28-29$ & $51.3(210)$ & $38.1(156)$ & $10.5(43)$ & 5.0 \\
\hline $30-31$ & $52.2(282)$ & $38.5(208)$ & $9.3(50)$ & 1.8 \\
\hline
\end{tabular}

Genotypes are given for the 1,354 infants in the study cohort. Neonatal mortality rates are reported for all the 1,773 infants who would have been in the cohort had dried blood spot samples been available - due to timing of sampling $83 \%$ were missing for children who died prior to 28 days' postnatal age.

Table 4. Estimates of association between UGT1A1*28 genotypes and time with supplementary oxygen $\geq 28$ days after birth for 1,343 preterm infants, born at 24-31 weeks' gestational age and still alive 28 days after birth

$\begin{array}{ll}\text { Cessation of supplementary oxygen, } & \begin{array}{l}\text { Duration of oxygen treatment, } \\ \text { difference days }(95 \% \mathrm{CI})\end{array} \\ \text { HR }(95 \% \mathrm{CI}) & \end{array}$

Common genotype Ref.

UGT1A $1 * 28$ heterozygotes $\quad 0.84(0.71$ to 1.00$)$

UGT1A1*28 homozygotes $\quad 0.70$ (0.50 to 0.99$)$
Ref.

$5.28(-0.18$ to 10.73$)$
10.55 ( -0.36 to 21.46$)$

All analyses were adjusted for birth year, sex and African race. HR were estimated by Cox regression and numeric difference in days with oxygen by generalized linear methods with pseudo values.

\section{Discussion}

\section{Key Results}

In this study, we examined the association between UGT1A $1 * 28$ genotypes and respiratory morbidity in a cohort of very preterm children. We found that UGT1A1*28 genotypes were associated with increased odds for any need of supplementary oxygen, a prolonged need of supplementary oxygen and an increased risk of $\mathrm{BPD}$ in preterm survivors. Preterm infants with
UGT1A1*28 genotypes had the same need for surfactant treatment as those with the common genotype.

HWE was unlikely for the cohort. This prompted a sensitivity analysis whereby we found that UGT1A1*28 genotypes were more prevalent in the extremely preterm infants than among the less preterm, the increased prevalence seemingly being associated with death prior to genotype sampling. In two tests of robustness, this association did not explain the primary estimates. 


\section{Strengths and Limitations}

Strengths

A major strength of our study is the availability of clinical data and genotypes covering more than a decade for a well-defined population. By combining the clinical information from two tertiary NICUs serving a specific geographical area with the biological data at the Danish Neonatal Screening Biobank, we were able to study the association between UGT1A $1 * 28$ genotypes and respiratory morbidity for a large cohort of very preterm infants.

\section{Limitations}

HWE was unlikely for the cohort. This implies one of the following: genotyping is flawed, population stratification is present or selection upon the tested genotype has taken place [22]. Our genotyping was performed at the same time as two control series (some 400 and 1,500 controls, respectively), and none of these showed evidence against HWE $[11,23]$. Furthermore, we tested a subsample in two different laboratories with two different methods and found no difference in genotypes.

The Danish population is genetically very homogeneous, and in a sensitivity test, ethnic heterogeneity failed to explain the lack of HWE. Thus, selection may be the most likely cause of the deviation from HWE. The study cohort was selected toward survivors, and UGT1A $1 * 28$ genotypes increased with decreasing gestational age and increasing mortality. It is possible that fetuses with the UGT1A $1 * 28$ genotypes are more prone to be born preterm, but our data may also suggest ex utero selection and an increased chance of survival in preterm infants with UGT1A $1 * 28$ genotypes.

As death and $\mathrm{BPD} /$ cessation of supplementary oxygen are competing outcomes, an association between UGT1A $1 * 28$ genotypes and survival could cause immortal person-time bias in our estimates [24]. A recommended approach to avoid immortal person-time bias is to exclude the immortal time from the denominator [24]. We did so in a test of robustness by only including risk time after 28 days' postnatal age, and the results were essentially unchanged. In addition, our results also remained essentially unchanged after stratification according to gestational age (a proxy variable for the risk of early death). Accordingly, immortal person-time bias is unlikely to explain our results.

We adjusted for African heritage due to a higher frequency of UGT1A $1 * 28$ genotypes and a lower neonatal morbidity in this population $[10,25]$. Few infants of African heritage were present in the cohort, and the adjustment made no difference to our results.

\section{Interpretation}

The UGT1A1 enzyme metabolizes a variety of other endogenous and exogenous substances apart from bilirubin [10]. Also, the association between UGT1A1*28 genotypes and plasma bilirubin is well established in adults, but no data for preterm infants exist. Thus, it is very possible that metabolites other than bilirubin may play a role in the association between UGT1A1*28 genotypes and preterm respiratory morbidity.

Morris et al. [5] published a large randomized trial of two thresholds of serum bilirubin for phototherapy in very preterm infants. In the secondary analyses, they found that a higher treatment threshold, and thus higher overall bilirubin levels, was associated with a higher risk of BPD. This is in accordance with our findings.

A decreased respiratory drive due to a direct effect on the central nervous system of higher levels of bilirubin may be one potential explanation of these findings. A previous study has shown an association between hyperbilirubinemia and the risk of apnea and bradycardia in preterm infants [26]. Furthermore, analyses from the trial by Morris et al. [5] showed neurotoxic effects from plasma bilirubin levels at higher treatment thresholds.

We found no association between UGT1A1*28 genotypes and the need of rescue surfactant in the very preterm infants. This is in line with data that suggest that UGT1A1 activity is very low in the preterm infant immediately after birth and, therefore, that no effect of the genotype in the first days of life is to be expected [27].

In preterm infants with birth weights of 501-750 g, Morris et al. [5] found that a high phototherapy treatment threshold (a high bilirubin level) was associated with increased survival. This could be in accordance with our observation of an increased frequency of UGT1A $1 * 28$ genotypes amongst the most preterm surviving infants if bilirubin is the causative metabolite in our study.

\section{Conclusion}

Compared to the common genotype, UGT1A1*28 genotypes were associated with an increased need of oxygen supplementation and risk of BPD in preterm newborns.

\section{Acknowledgment}

The study was supported by a grant from the Danish Council for Independent Research (DFF-1331-00297B). 


\section{References}

1 Sweet DG, Carnielli V, Greisen G, Hallman M, Ozek E, Plavka R, Saugstad OD, Simeoni U, Speer CP, Vento M, Halliday HL: European consensus guidelines on the management of neonatal respiratory distress syndrome in preterm infants - 2013 update. Neonatology 2013;103:353-368.

2 Saugstad OD, Aune D: Optimal oxygenation of extremely low birth weight infants: a metaanalysis and systematic review of the oxygen saturation target studies. Neonatology 2014; 105:55-63.

3 Stocker R, Yamamoto $\mathrm{Y}$, McDonagh AF, Glazer AN, Ames BN: Bilirubin is an antioxidant of possible physiological importance. Science 1987;235:1043-1046.

4 Benaron DA, Bowen FW: Variation of initial serum bilirubin rise in newborn infants with type of illness. Lancet 1991;338:78-81.

5 Morris BH, Oh W, Tyson JE, et al: Aggressive vs. conservative phototherapy for infants with extremely low birth weight. N Engl J Med 2008;359:1885-1896

6 Zhu J, Xu Y, Zhang G, Wu M, Du L: Total serum bilirubin level in umbilical cord blood and respiratory distress syndrome in very low birth weight infants. J Perinat Med 2011;40:91-95.

7 Bosma PJ, Chowdhury JR, Bakker C, Gantla S, de BA, Oostra BA, Lindhout D, Tytgat GN, Jansen PL, Oude Elferink RP: The genetic basis of the reduced expression of bilirubin UDP-glucuronosyltransferase 1 in Gilbert's syndrome. N Engl J Med 1995;333:1171-1175.

8 Bosma PJ, Seppen J, Goldhoorn B, Bakker C, Oude Elferink RP, Chowdhury JR, Chowdhury NR, Jansen PL: Bilirubin UDP-glucuronosyltransferase 1 is the only relevant bilirubin glucuronidating isoform in man. J Biol Chem 1994;269:17960-17964.

9 Stender S, Frikke-Schmidt R, Nordestgaard BG, Grande P, Tybjaerg-Hansen A: Genetically elevated bilirubin and risk of ischaemic heart disease: three Mendelian randomization studies and a meta-analysis. J Intern Med 2013;273:59-68.
10 Strassburg CP: Gilbert-Meulengracht's syndrome and pharmacogenetics: is jaundice just the tip of the iceberg? Drug Metab Rev 2010; 42:168-181.

11 Petersen JP, Henriksen TB, Hollegaard MV, Vandborg PK, Hougaard DM, ThorlaciusUssing O, Ebbesen F: Extreme neonatal hyperbilirubinemia and a specific genotype: a population-based case-control study. Pediatrics 2014;134:510-515.

12 Roy-Chowdhury N, Deocharan B, Bejjanki HR, Roy-Chowdhury J, Koliopoulos C, Petmezaki S, Valaes T: Presence of the genetic marker for gilbert syndrome is associated with increased level and duration of neonatal jaundice. Acta Paediatr 2002;91:100-101.

13 Kaplan M, Renbaum P, Hammerman C, Vreman HJ, Wong RJ, Stevenson DK: Heme oxygenase-1 promoter polymorphisms and neonatal jaundice. Neonatology 2014;106:323329.

14 Katan MB: Apolipoprotein E isoforms, serum cholesterol, and cancer. Lancet 1986;1:507508.

15 Verder H, Ebbesen F, Fenger-Gron J, Henriksen TB, Andreasson B, Bender L, Bertelsen A, Bjorklund LJ, Dahl M, Esberg G, Eschen C, Hovring M, Kreft A, Kroner J, Lundberg F, Pedersen P, Reinholdt J, Stanchev H: Early surfactant guided by lamellar body counts on gastric aspirate in very preterm infants. Neonatology 2013;104:116-122.

16 Andersson S, Petersen J, Brink HT, Ebbesen F: The Danish neonatal clinical database is valuable for epidemiologic research in respiratory disease in preterm infants. BMC Pediatr 2014;14:47.

17 Pedersen CB, Gotzsche H, Moller JO, Mortensen PB: The Danish civil registration system. A cohort of eight million persons. Dan Med Bull 2006;53:441-449.

18 Norgaard-Pedersen B, Hougaard DM: Storage policies and use of the Danish newborn screening biobank. J Inherit Metab Dis 2007; 30:530-536.
19 Hollegaard MV, Thorsen P, Norgaard-Pedersen B, Hougaard DM: Genotyping whole-genome-amplified DNA from 3- to 25-year-old neonatal dried blood spot samples with reference to fresh genomic DNA. Electrophoresis 2009;30:2532-2535.

20 Saeki M, Saito Y, Jinno H, Tohkin M, Kurose K, Kaniwa N, Komamura K, Ueno K, Kamakura S, Kitakaze M, Ozawa S, Sawada J: Comprehensive UGT1A1 genotyping in a Japanese population by pyrosequencing. Clin Chem 2003;49:1182-1185.

21 Parner ET, Andersen PK: Regression analysis of censored data using pseudo-observations. Stata J 2010;10:408-422.

22 Guo SW, Thompson EA: Performing the exact test of Hardy-Weinberg proportion for multiple alleles. Biometrics 1992;48:361-372.

23 Petersen JP, Overvad K, Hollegaard MV, Ebbesen F, Henriksen TB, Thorlacius-Ussing O, Hougaard DM, Schroder H: UGT1A1*28 polymorphism and acute lymphoblastic leukemia in children: a Danish case-control study. Pediatr Res 2014;76:459-463.

24 Rothman KJ, Greenland S: Cohort studies; in Rothman KJ, Greenland S, Lash TL (eds): Modern Epidemiology, ed 3. Philadelphia, Lippincott, Williams \& Wilkins, 2008, pp 106-107.

25 Kavvadia V, Greenough A, Dimitriou G, Hooper R: Influence of ethnic origin on respiratory distress syndrome in very premature infants. Arch Dis Child Fetal Neonatal Ed 1998;78:F25-F28.

26 Amin SB, Charafeddine L, Guillet R: Transient bilirubin encephalopathy and apnea of prematurity in 28 to 32 weeks gestational age infants. J Perinatol 2005;25:386-390.

27 Watchko JF, Lin Z, Clark RH, Kelleher AS, Walker MW, Spitzer AR: Complex multifactorial nature of significant hyperbilirubinemia in neonates. Pediatrics 2009;124:e868e877. 